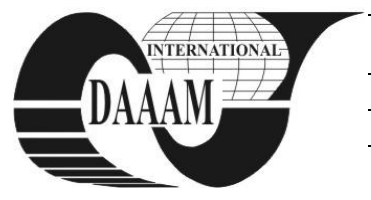

\title{
ROBOTAS - DEVELOPMENT OF A MOBILE ROBOT FOR SMALL GOODS TRANSPORT
}

\author{
PRODROMOU, D[imitrios]
}

\begin{abstract}
This paper deals with the development of a robot prototype, which carries goods of a person in a supermarket using low-cost sensors, like the Kinect camera, in a real environment. From the combination of different tracking methods, two versions of the overall system were developed: version 1 based on $3 D$ point cloud and a special template matcher and an improved version 2 based on depth image and a special particle filter. Experiments for unique identification and distance calculation with a Bluetooth system were carried out but ultimately proved too inaccurate with the given hardware. Under constant lightning conditions, the target person could be found with a high confidence. For unsteady lighting conditions, color information distortions by the camera resulted in calculation errors and in wrong match results. In summary, the prototype already provided very good results at this early stage
\end{abstract}

Key words: OpenCV, robot operating system, kinect, robotic shopping card, wireless systems

\section{INTRODUCTION}

Some mobile robots, such as vacuum cleaners and lawn mowers, have already made their first steps towards household robotics. Mostly without "eyes", they move almost blind in their environment and react to collisions or approaches to walls or obstacles. What is perfectly adequate for the household, would not be enough for a shopping cart in a supermarket. In this environment, the robot has to deal with much more influences and problems, than a simple vacuum cleaner could handle.

\section{PROBLEM AND TASK}

The basic idea of this work is to develop a prototype of a fully automated shopping cart, available to a person during their stay at a supermarket. A typical office scene was used as the test environment for initial experiments.

The robot should detect the person in an initialization phase, learn his/her appearance and follow her across the room. Obstacles and other people which are near the target person or in between the robot and the target person will not be recognized as targets. Optionally, different wireless technologies are compared and an implementation into the entire system should be considered.

\section{STATE OF THE ART}

According to the only available source, a YouTube-video, the mobile robot platform (Cytron Technologies, 2009) could follow a person through a shopping mall, scanning items and waiting for orders. However, there is no scientific proof of the real functionality of the robot.

The multi-sensor human tracking system was a more promising student project at the School of Computer Science and Engineering (University of New South Wales, 2008). The project implied the following: the robot was tracking and following a person through other people and obstacles, using a normal webcam and a laser tracker. The system provided good results at the end of the project, while some parts, such as avoiding and driving around obstacles, were not implemented.

The mobile robot platform followed the closest person using a laser scanner. In addition to that, the second system used a webcam to find the target person after losing her. A unique identification achieved by the use of a color camera and image processing algorithms as provided in our system, led to a much better recognition and tracking result of the target person.

\section{PRACTICAL IMPLEMENTATION}

We use ROS (Robot Operating System) as the software framework to communicate with the robot and the Kinect camera. The information received from the camera images are processed by the OpenCV vision library, which includes all functions for detecting and tracking a person in an image.

The hardware consists of a Pioneer P3-DX mobile robot which largely met all project requirements. Furthermore the chosen Microsoft Kinect camera provides very good pictures and a depth image at a low purchase price.

\subsection{Person Detection and Tracking}

In version 1 of the overall system a combination of the $3 \mathrm{D}$ point cloud and a special FastTemplateMatcher (Georgiou, 2009) was used. As already mentioned, the system needs a way to determine the distance to the target. ROS provides a special point cloud library for filtering and editing of 3D point clouds. With the use of those functions the unnecessary background can be deleted and only the target person is captured in 20 initialization pictures. The FastTemplateMatcher uses those pictures to find and track the person and delivers their position to the robot.
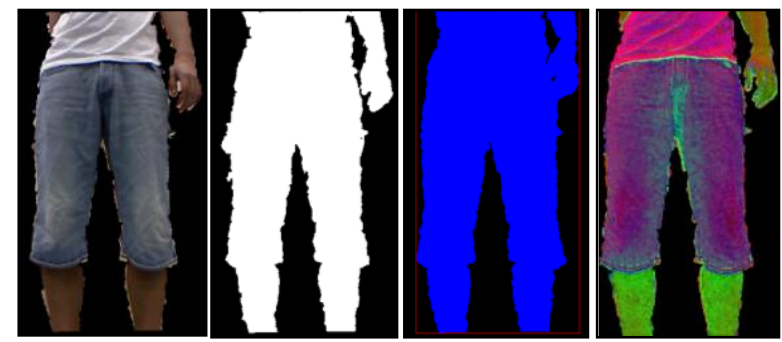

Fig. 1. Different views of version 2 of the overall system: (from left to right) filtered RGB image, filtered depth image, captured person with the Open CV FloodFill function, filtered HSV image

Version 2 (Fig. 1) of the overall system, however, was designed with the faster and less time consuming depth image and a specially adapted particle filter. In addition to the 3D point cloud, the background was deleted and the target person was captured into an initialization histogram. From the 
combination of the $2 \mathrm{D}$ histogram comparison function and $3 \mathrm{D}$ distance calculation, the particle filter continuously monitors the probability that the current person is also the target person.

\subsection{Wireless systems}

The use of identification chips would precisely tie up a robot to a target person and would also allow an additional distance measurement. Different technologies have been theoretically analyzed and compared. Grossmann et al. (2007) already measured various parameters of the ZigBee system compared to the distance and came to relatively good results. Bluetooth provides a similar wireless technology with the advantage of being widely available. The test setup consisted of a Bluetooth capable mobile phone (LG P990), a laptop with Bluetooth 3.0 and a measured length of $20 \mathrm{~m}$. First, the measured values (Link Quality (LQ), Received Signal Strength Indicator (RSSI) and Transmit Power Level (TPL)) at the starting position of $0 \mathrm{~m}$ were recorded. Then, $1 \mathrm{~m}$ measuring steps were repeated until the entire $10 \mathrm{~m}$ route was covered. Finally, a last measurement at $25 \mathrm{~m}$ was performed.

\section{RESULTS}

For the experiments with the Bluetooth system, the Linux hcitool was used. With this, Bluetooth devices can be identified and different parameters can be measured. The important parameters used for this experiment were the LQ, the RSSI and the TPL (Farahani, 2008).

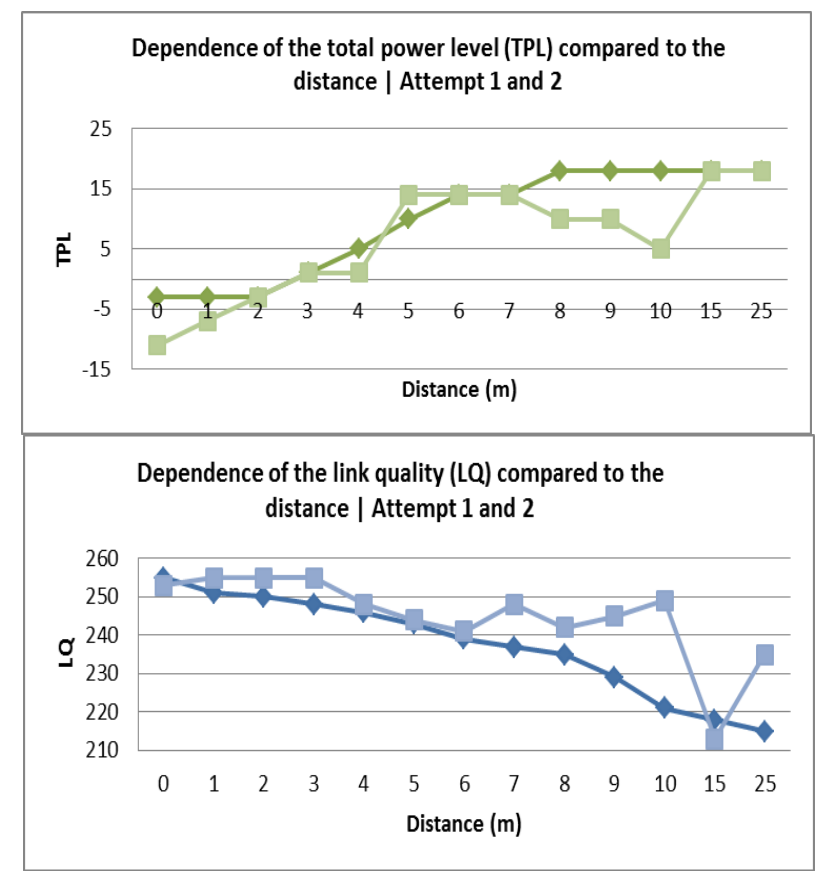

Fig. 2. Results of the LQ and TPLs from Experiment 1 and 2

The results of the two Bluetooth experiments are shown in Figure 2. The TPL had a greater overall variation than the LQ. The RSSI value had no significant results. Furthermore, the measurements had a certain lag while adjusting to a new distance, which may have further influenced the results. Another experiment with an existing data connection between laptop and mobile phone achieved much better results, but was also very battery consuming. In summary, the results were not significant enough to calculate the position of a target person. Sophisticated hardware with controllable TPL settings would possible lead to better results.

\subsection{Experiments with versions 1 and 2 of the overall system}

Since version 1 of the overall system using the 3D point cloud and a modified FastTemplateMatcher was time and processor consuming, no sufficiently good recognition rates could be achieved. Recognition speed could be increased remarkably by combining the depth image with the specially modified particle filter in version 2 of the overall system.

Recognition and tracking of a person with long blue jeans, a colorful skirt and short blue jeans was tested in each experiment (Fig. 3 left). In addition to that, a second person, used to distract the system, would appear in the image (Fig. 3 middle and right). In constant lighting conditions all experiments with version 2 of the overall system led to good recognition results. However, in unsteady lighting conditions, problems with the internal white balance and color adjustment of the Kinect camera led to false-color images and additionally to that, to false match results with other objects such as cabinets or boxes.
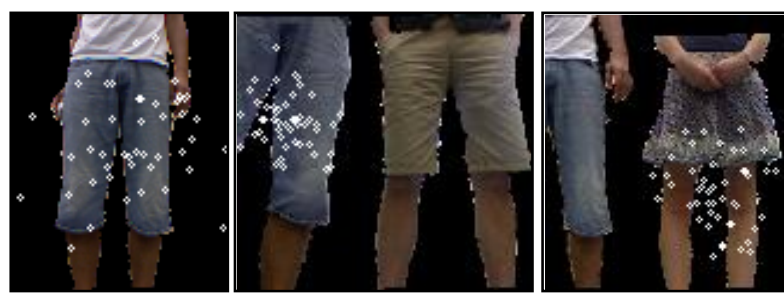

Fig. 3. Different views of the particle filter left: found target, center and right: target person found despite second person in the picture

\section{CONCLUSION AND FUTURE REASEARCH}

In summary, the development of the prototype could be completed successfully. The person was successfully detected in the image and finally tracked in a real environment. Despite getting wrong results caused by lightning conditions, the overall system still offered a robust detection of the target person.

The potential for expansion and improvements is very large. Furthermore, version 2 of the overall system can be extended with new detection algorithms and better identification methods to track the target person. Especially the implementation of better wireless identification method and the use of sophisticated wireless hardware should be a major part in future projects. Much better identification of the target person can be performed with that. For this purpose, a better color camera can be used to capture the target person histogram. In particular, the configuration of the white balance and color adjustments would lead to minimization of the distortions during unsteady lightning conditions.

\section{REFERENCES}

Farahani S. (2008). ZigBee wireless networks and transceivers, Elsevier Information Systems GmbH, 978-0750683937, Frankfurt

Georgiou T. (2011). Fast Match Template, Available from: opencv.willowgarage.com/wiki/FastMatchTemplate Accessed: 2011-08-12

Grossmann R., Blumenthal J., Golatowski F., Timmermann D., (2007). Localization in ZigBee-based wireless sensor networks, Available from: http://www.lomsitea.org/publications/LocalizationZigbee.pdf Accessed: 2011-08-12

Cytron Technologies (2009). Mobile Robot Platform, Available from: http://tutorial.cytron.com.my/2009/05/26/mobilerobot-platform/ Accessed: 2011-08-12

University of New South Wales (2008). Multi-sensor human tracking (final report), Available from: http://cgi.cse.unsw.edu.au/ cs4411/wiki/index.php?title=M ulti-sensor_human_tracking_(final_report) Accessed: 2011$08-12$ 